\title{
Einstein's much delayed little joke
}

\section{When Einstein invented the cosmological constant in 1917, and promptly disowned it, he cannot have known that the concept would be bothering his successors more than 70 years later.}

EINSTEIN had very few reasons to be ashamed of any of his work, but his invention of what is called the cosmological constant seems to have been one of them. And it is a curious business. Having developed the theory of gravitation called the General Theory of Relativity, Einstein embarked in 1917 on an attempt to apply his equations to the Universe as a whole. On the principle that the motions of the most distant objects in the Universe - the first galaxies to be recognized as such were being measured at the time are uniformly much less than the velocity of light, Einstein set out to find solutions of his equations that might be taken to represent a static Universe, but could not.

So, with a daring that must have seemed natural to one who had just used pure thought to put the then laws of physics on a rational foundation, he invented the cosmological constant and promptly reached a solution of the desired form - a spherical fixed universe filled with matter of uniform density. But within a few months, Einstein had withdrawn the notion of the cosmological constant.

Legend has it that Einstein's disillusion sprang from Hubble's discovery that the Universe is expanding (so that the search for a static solution would have seemed a wild goose chase), but Steven Weinberg, writing from the University of Texas at Austin, tells a different story in an elegant review article just published (Rev. Mod. Phys. 61, 1; 1989).

Serious accounts of the dilemma make it plain that the difficulty that gave Einstein pause was de Sitter's demonstration a few months later that there is a static solution of Einstein's equations with the uncomfortable property that the Universe it represents is entirely devoid of matter. This may have seemed a body blow, for it cast doubt on Einstein's guiding principle that mechanical inertia is a consequence of the distribution of mass within the Universe.

In the event, as Weinberg puts it, the need for the cosmological constant was entirely undermined by the emergence in the 1920 s of the family of cosmologies due to A. Friedman, which allowed for an expanding or a contracting Universe simply by the appropriate choice of a parameter. Yet the irony remains that, despite Einstein's disavowal of his own invention, the question of whether the cosmological constant exists, and is different from zero, and of what precisely it may mean, is more vividly alive now than sixty years ago.

The bare bones of the issue are easily understood. Einstein's picture of the geometry of space-time turns on the set of 16 coefficients defining the relativistic equivalent of distance (or time) by means of a quadratic form called a metric; only 10 of these coefficients are independent, because the form is symmetric, but general relativity differs from special relativity in that the coefficients are functions of position. The original field equations relate these coefficients at any place-time to the curvature at the same place-time, which is a function of the same coefficients. (This is why the equations are not linear and are thus not soluble systematically.)

The physics of general relativity is embodied in the way in which the metric coefficients are related to the density of energy (which includes mass) and momentum in the system. The effect of Einstein's introduction of his cosmological constant is dimensionally and in every other way equivalent to the arbitrary addition of energy and momentum to empty space, in quantities determined by the size of the constant. That explains why the constant should have turned an expanding into a static universe. In the distant early 1920 s, it is understandable that Einstein should first have taken the liberty of introducing the cosmological constant and then, when it seemed no longer necessary, the liberty of discarding it.

Quantum mechanics has put a stop to that. That is Weinberg's starting point. The essence of his case is that, whatever Einstein's pros and cons may have been, nobody can now hide from the truth that even empty space is filled with energy to say the least of it, the zero-point energy of all the oscillators corresponding to radiation of different frequency with which even a vacuum must be filled but, more tangibly perhaps, because pairs of particles (such as electrons and positrons) can be created out of nothing anywhere and any time by what are called the fluctuations of the vacuum.

From this observation springs the dilemma that now perplexes people. If the effect of the unavoidable fluctuations of the vacuum are logically equivalent to a cosmological constant different from zero, and if the observation of the real Universe shows that Einstein's cosmological constant is unnecessary, then the underlying equations of general relativity must include a 'cosmological constant' whose value is exactly cancelled by that simulated by the fluctuations of the vacuum. And the cancellation must be extraordinarily exact.

The net effect of the 'real' and 'simulated' constants can be related to Hubble's constant, which determines the rate at which the Universe is expanding, and which serves as an upper bound, while the value of the constant simulated by vacuum fluctuations can be estimated from the properties of quantum systems. Weinberg concludes that the two lines of argument are consistent only if the true and simulated values of the cosmological constant cancel each other out to 118 decimal places (although, on another estimate of the quantum fluctuations, he estimates that the cancellation may extend to only 41 decimal places). For practical purposes, this is simply another way of saying that the numbers are equal.

Weinberg's objective is to consider how the extraordinary coincidence implied by these estimates can be rationally explained. He flirts with several possibilities, only to dismiss them. The supersymmetric world in which fermions (electrons, say) and bosons (say photons) are identical might arrange for the cancellation if the supersymmetric world were like the real world (which it is not). The superstring theory of particle fields (in which six of ten dimensions are made to disappear, giving entities which are elastic strings in ten dimensions, the properties of the particles we know in only four) appears to have offered hope at first, but to have proved a disappointment.

The anthropic view, that people are able to observe the stars and galaxies only if the Universe in which they find themselves is one in which the cancellation is exact is plainly seductive, but Weinberg eventually dismisses it in each of half a dozen varieties. His own clear favourite is that the cancellation will spring naturally from the attempts being made to develop a quantum theory of gravity, but there may be as many opinions as there are people qualified to write about the problem.

Meanwhile, it is clear that Einstein was even more perceptive than he knew. It cannot be often that the notions a person discards turn out to be crucial to a conundrum that perplexes his successors the best part of a century later. John Maddox 